\title{
Protocolo de intervenção cinesioterapêutica com exergames para força muscular e equilíbrio em idosos - uma revisão sistemática da literatura
}

Kinesiotherapeutic intervention protocol with exergames for muscle strength and balance in elderly - a systematic literature review

Protocolo de intervención kinesioterapéutica con ejercicios para la fuerza y el equilibrio muscular en ancianos - revisión sistemática de la literatura

Fabrízzio Martin Pelle Perez ORCID: https://orcid.org/0000-0001-5069-4256

Universidade de Passo Fundo, Brasil E-mail: fabrizziopelle@gmail.com

Patrícia Paula Bazzanello Henrique ORCID: https://orcid.org/0000-0003-4224-0419 Universidade de Passo Fundo, Brasil E-mail: patriciabazzanello@hotmail.com

Ana Carolina Bertoletti De Marchi ORCID: https://orcid.org/0000-0002-7704-3119 Universidade de Passo Fundo, Brasil E-mail: carolina@upf.br

\begin{abstract}
Resumo
Objetivos: Mapear e discutir os diferentes protocolos de intervenção cinesioterapêutica com exergames para força muscular e equilíbrio em idosos e investigar métodos de avaliação de equilíbrio, força muscular e o tempo de intervenção. Materiais e Métodos: Realizamos uma revisão sistemática da literatura entre agosto de 2019 e janeiro de 2020 na interface da Biblioteca Virtual em Saúde, Springer, ScienceDirect e Pedro. A busca considerou estudos publicados até dezembro de 2019, sem restrição de idioma. Um total de 408 registros foram identificados. No processo de seleção, 17 estudos atenderam aos critérios de inclusão para análise. Resultados: Dos 17 estudos analisados, cinco demonstraram que o protocolo de exercícios cinesioterapêuticos com exergames foi eficaz na melhora do equilíbrio e força muscular de idosos. Sete estudos demonstraram a eficácia dos protocolos convencionais juntamente aos protocolos com exergames. Cinco estudos analisados mostraram a eficácia do exergame quando comparado com o protocolo convencional. A escala de equilíbrio de Berg e o teste TUG, foram as avaliações de equilíbrio mais utilizada e apenas um estudo relatou detalhadamente a forma de avaliação da força muscular. A maioria dos estudos realizaram o protocolo de intervenção num período de 8 a 12 semanas. Conclusão: Exergame associado a um protocolo de intervenção cinesioterapêutica ou exercícios físicos melhora a força muscular e equilíbrio em idosos.
\end{abstract}

Palavras-chave: Exergame; Envelhecimento; Fisioterapia; Força muscular; Equilíbrio.

\begin{abstract}
Objectives: To map and discuss the different kinesiotherapeutic intervention protocols with exergames for muscle strength and balance in the elderly and to investigate methods for assessing balance, muscle strength and intervention time. Materials and Methods: We performed a systematic literature review between August 2019 and January 2020 at the Virtual Health Library interface, Springer, ScienceDirect and Pedro. The search considered studies published until December 2019, without language restrictions. A total of 408 records were identified. In the selection process, 17 studies met the inclusion criteria for analysis. Results: Of the 17 studies analyzed, five demonstrated that the kinesiotherapeutic exercise protocol with exergames was effective in improving balance and muscle strength in the elderly. Seven studies demonstrated the effectiveness of conventional protocols alongside exergame protocols. Five studies analyzed showed the effectiveness of the exergame when compared to the conventional protocol. The Berg balance scale and the TUG test were the most used balance assessments and only one study reported in detail how muscle strength was assessed. Most studies performed the intervention protocol over a period of 8 to 12 weeks. Conclusion: Exergame associated with a kinesiotherapy intervention protocol or physical exercise improves muscle strength and balance in the elderly.
\end{abstract}

Keywords: Exergame; Aging; Physiotherapy; Muscle strength; Balance. 


\begin{abstract}
Resumen
Objetivos: Mapear y discutir los diferentes protocolos de intervención kinesioterapéutica con exergames para la fuerza muscular y el equilibrio en el anciano e investigar métodos para evaluar el equilibrio, la fuerza muscular y el tiempo de intervención. Materiales y métodos: Realizamos una revisión bibliográfica sistemática entre agosto de 2019 y enero de 2020 en la interfaz de la Biblioteca Virtual en Salud, Springer, ScienceDirect y Pedro. La búsqueda consideró estudios publicados hasta diciembre de 2019, sin restricciones de idioma. Se identificaron un total de 408 registros. En el proceso de selección, 17 estudios cumplieron los criterios de inclusión para el análisis. Resultados: De los 17 estudios analizados, cinco demostraron que el protocolo de ejercicio kinesioterapéutico con exergames fue eficaz para mejorar el equilibrio y la fuerza muscular en los ancianos. Siete estudios demostraron la efectividad de los protocolos convencionales junto con los protocolos de exergame. Cinco estudios analizados mostraron la efectividad del exergame en comparación con el protocolo convencional. La escala de equilibrio de Berg y la prueba TUG fueron las evaluaciones de equilibrio más utilizadas y solo un estudio informó en detalle cómo se evaluó la fuerza muscular. La mayoría de los estudios realizaron el protocolo de intervención durante un período de 8 a 12 semanas. Conclusión: el juego de ejercicios asociado a un protocolo de intervención de kinesioterapia o ejercicio físico mejora la fuerza muscular y el equilibrio en los ancianos.
\end{abstract}

Palabras clave: Exergames; Envejecimiento; Fisioterapia; Fuerza muscular; Equilíbrio.

\title{
1. Introdução
}

O envelhecimento, apesar de ser um processo natural, é responsável por algumas alterações fisiológicas, como diminuição da força muscular e equilíbrio (Davini, 2003). Tais riscos podem ser amenizados por meio da realização de exercícios físicos ou cinesioterapêuticos regularmente (Tomocki et al., 2016; Manlapaz et al., 2017).

Um programa de exercícios cinesioterapêuticos para ganho de equilíbrio e força muscular pode manter ou recuperar determinadas funções sensoriomotoras e cardiovasculares (Barnabera et al., 2014), tornando-se importante para auxiliar na melhora dos aspectos funcionais do idoso. O desafio está em propor uma prática fisioterapêutica inovadora, com resultados satisfatórios e que motive os idosos (Mendonça et al., 2018). Nesse cenário, novos modelos de assistência ao idoso precisam ser propostos e avaliados constantemente (Barnabera et al., 2014), como os exercícios da reabilitação virtual, que se popularizaram na última década como uma opção inovadora nos programas de reabilitação (Agmon et al., 2011). A reabilitação virtual pode ser realizada com o uso de exergames, jogos que utilizam dispositivos de interação física com o usuário como forma de exercício (Treml et al., 2013). Estes jogos buscam incentivar o uso de movimentos corporais para interação com o ambiente virtual (Baracho et al., 2012).

A realização de exercícios cinesioterapêuticos com o uso de reabilitação virtual vem evoluindo e se aprimorando. O uso de jogos faz com que os pacientes fiquem mais motivados e satisfeitos durante as sessões e tenham maior aderência ao tratamento. Os jogos fornecem uma natureza estimulante, integrativa e o ambiente torna-se enriquecido e com maior motivação para o aprendizado de habilidades motoras (Vilela et al., 2017). Eles já tiveram sua comprovação comprovada em pacientes pós-AVE (Henrique et a., 2019), idosos saudáveis (Brandão et al., 2017), na capacidade funcional (Mussato et al., 2012), equilíbrio (Ku et al., 2018; Stanmore et al., 2019), e força muscular (Padala et al., 2017; Cordeiro et al., 2019).

O número crescente de exergames comerciais ou desenvolvidos revela um panorama de diferentes aplicações para reabilitação motora. Além de demonstrarem dinamismo e heterogeneidade, os estudos de intervenção para avaliar os efeitos dos exergames apresentam distintas técnicas de coleta de dados, instrumentos, número de participantes, tempo de intervenção e especificidade a ser reabilitada. Adicionalmente, comparando ao tratamento fisioterapêutico usual, os exergames apresentam melhorias na adesão ao tratamento e redução no número de sessões de tratamento (Wu et al., 2015).

Neste sentido, com o objetivo de buscar evidências da eficácia do uso de exergames no tratamento cinesioterapêutico, nós revisamos sistematicamente a literatura para identificar e analisar estudos que abordaram os efeitos de um programa de intervenção cinesioterapêutica com exergame sobre a força muscular e equilíbrio. Este estudo busca investigar os métodos de avaliação do equilíbrio e força muscular utilizados e o tempo de intervenção observado nas respectivas pesquisas selecionadas. 


\section{Metodologia}

Esta pesquisa apresenta uma Revisão Sistemática da Literatura (RSL), realizada entre agosto de 2019 e janeiro de 2020. Uma revisão sistemática da literatura é caracterizada pela revisão de uma pergunta formulada de forma clara e que utiliza métodos sistemáticos para selecionar, identificar e analisar pesquisas, com posterior coleta e análise dos dados selecionados que serão incluídos na respectiva revisão (Moher et al., 2009).

\subsection{Questões Chave}

Pergunta Chave 1. Quais são os métodos de avaliação do equilíbrio e da força muscular usados?

Pergunta Chave 2. Qual o tempo total de intervenção utilizados nos estudos?

Pergunta Chave 3. Quais os dispositivos utilizados?

Pergunta Chave 4. Quais estudos relacionaram as escalas de equilíbrio com protocolos com e sem o uso de exergames?

\subsection{Estratégia de busca}

Consultamos sinônimos no serviço de vocabulário estruturado DeCS (Nlm, 2019). Pela estratégia PICO, a string de pesquisa foi: (Game or Exergame or Video game) and (Kynesiotherapy or Physiotherapy or Physical activity or Physical exercise or Physical therapy) and (Senoirs or Elderly or Older or Aging) and (Muscle strength or Muscle action or Balance or Stability or Muscle or Strength).

Este estudo utilizou bases multidisciplinares das Ciências da Saúde:

- Biblioteca Virtual em Saúde (BVS), na qual continham as bases MEDLINE e LILACS;

- Springer International Publisher Science, Technology, Medicine (Springer);

- ScienceDirect;

- Physiotherapy Evidence Data Base (PEDro).

\subsection{Critérios de Eligibilidade (CE)}

Os critérios de elegibilidade para a inclusão dos resultados estão descritos a seguir:

- CE1. Empregar o exergame combinado a um protocolo de intervenção cinesioterapêutica, atividade/exercício físico em idosos saudáveis; - CE2. Usar o exergame na busca de respostas sobre força muscular e equilíbrio; - CE3. As intervenções apresentadas deverão ser num público de idosos hígidos.

\subsection{Processo de seleção de estudos}

O processo de seleção dos estudos foi estruturado em três etapas: Fase 1 - Identificação das pesquisas nas bases de dados selecionadas, descritas anteriormente; Fase 2 - Leitura do título e resumo dos estudos encontrados nas bases de dados; Fase 3 - Analise dos estudos através da leitura completa para adequar aos critérios de elegibilidade.

Três pesquisadores realizaram o processo de seleção de forma simultânea e independente, arranjando os artigos com o software Mendeley (Lo Russo et al., 2013). 
Figura 1. Diagrama de fluxo de processo de seleção para estudos incluídos na revisão sistemática - adaptação do diagrama PRISMA (Moher et al., 2009).
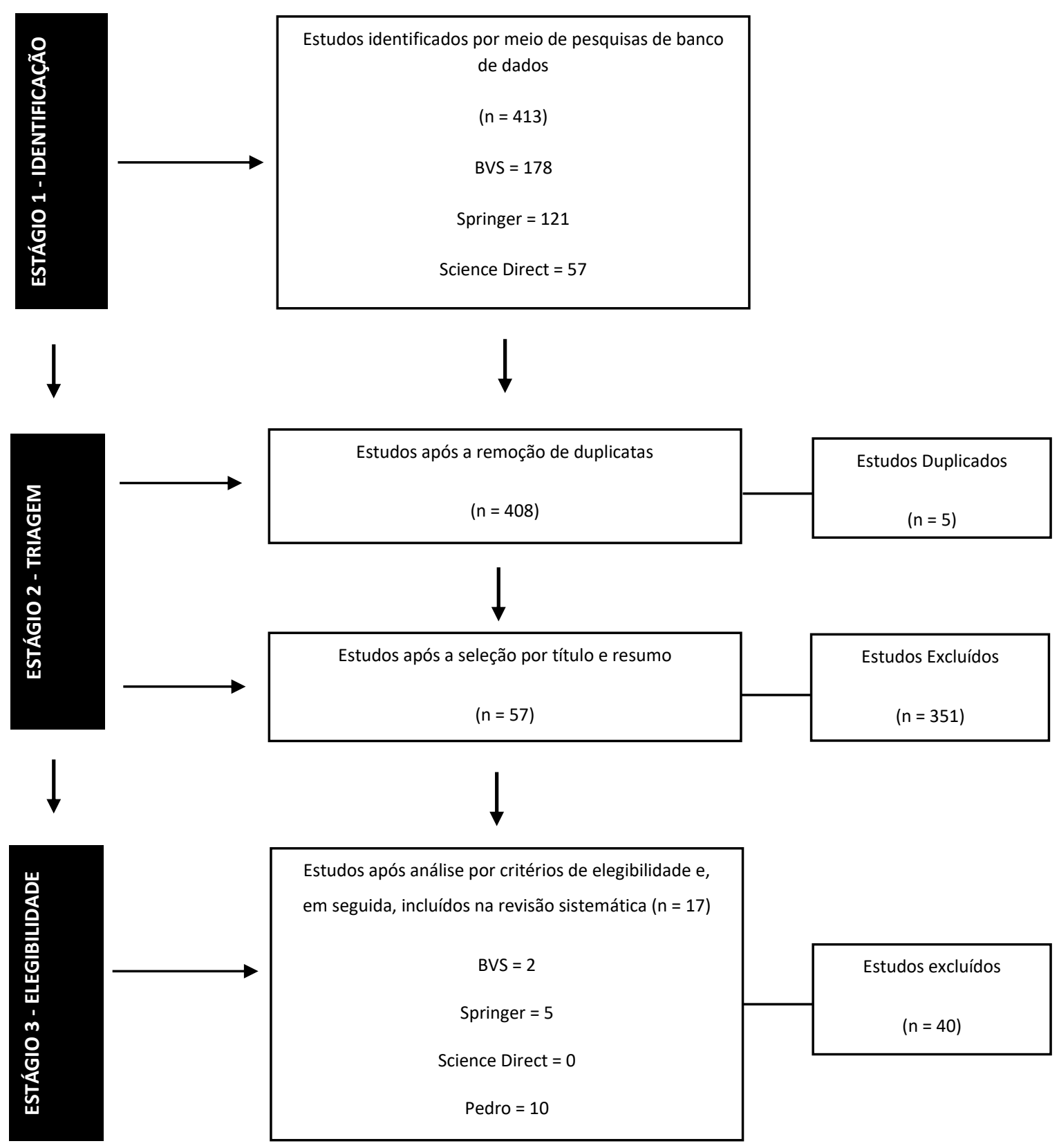

Fonte: Autores.

Uma variedade de medidas de resultados foram utilizados para mensurar os efeitos dos protocolos através de exergames e protocolos convencionais. Os testes de equilíbrio mais comumente utilizados foram, Escala de Equilíbrio de Berg (EEB), com 10 estudos (Agmon et al., 2011; Treml et al., 2013; Ku et al., 2018; Stanmore et al., 2019; Padala et al., 2017; Janssen et al., 2013; Sato et al., 2015; Whyatt et al., 2015; Bieryla, 2012; Bateni, 2012). O teste Time Up And Go (TUG) foi observado em 5 estudos (Ku et al., 2018; Bieryla, 2013; Schoene et al., 2012; Park et al., 2015; Singh et al., 2013). O teste de 
pisar foi observado em apenas um estudo (Whyatt et al., 2015) e o teste de Tinetti também em apenas um estudo (Sato et al., 2015) conforme pode ser visualizado no Quadro 1.

Em relação a força muscular, pode-se observar que 8 artigos fizeram uma investigação sobre a referida avaliação (Quadro 1).

Quanto às ferramentas utilizadas nas intervenções, o sistema Nintendo Wii foi utilizado em onze estudos, dois estudos utilizaram o sistema Microsoft Kinect e nas ferramentas X-Box, 3D-ARS, DDR-Stepmania foram utilizados em apenas um estudo para cada ferramenta. Nas pesquisas selecionadas, pode-se observar que doze estudos realizaram dois tipos de protocolos. Um protocolo de intervenção foi realizado através de exercícios convencionais ou cinesioterapêuticos e compararam com um protocolo de intervenção associado ao uso de exergames.

Todos os artigos selecionados para a respectiva revisão sistemática caracterizam-se por serem jogos comerciais e não observou-se informações a respeito de jogos desenvolvidos (Quadro 1).

\section{Resultados}

Até janeiro de 2020, foram identificados 413 artigos nas bases de dados pesquisadas, com base nas estratégias adotadas. Após a realização das fases 1 e 2, 356 artigos foram excluídos por apresentarem duplicação e por não apresentarem afinidade com o assunto e com os critérios de elegibilidade. Com a leitura completa dos estudos $(n=57)$ e de acordo com critérios já descritos, foram excluídos 40 estudos, resultando em 17 pesquisas incluídas nessa revisão sistemática (Figura 1).

De acordo com os estudos selecionados e o ano de publicação, a maioria dos trabalhos selecionados encontra-se no ano de 2013, com seis artigos, totalizando 35,29\%. Com três pesquisas encontradas, o ano de 2015 apresentou 17,65\% na relação dos trabalhos selecionados. Os artigos mais recentes selecionados foram quatro, um estudo em 2017 e outro em 2018 e no ano de 2019 foram selecionados dois artigos, totalizando 23,52\% das pesquisas selecionadas (Quadro 1).

Uma variedade de medidas de resultados foi utilizada para mensurar os efeitos dos protocolos através de exergames e protocolos convencionais. Os testes de equilíbrio mais comumente utilizados foram, Escala de Equilíbrio de Berg (EEB), com 10 estudos (Agmon et al., 2011; Treml et al., 2013; Ku et al., 2018; Stanmore et al., 2019; Padala et al., 2017; Janssen et al., 2013; Sato et al., 2015; Whyatt et al., 2015; Bieryla, 2012; Bateni, 2012). O teste Time Up And Go (TUG) foi observado em 5 estudos (Ku et al., 2018; Bieryla, 2013; Schoene et al., 2012; Park et al., 2015; Singh et al., 2013). O teste de pisar foi observado em apenas um estudo (Whyatt et al., 2015) e o teste de Tinetti também em apenas um estudo (Sato et al., 2015) conforme pode ser visualizado no Quadro 1.

Em relação a força muscular, pode-se observar que 8 artigos fizeram uma investigação sobre a referida avaliação (Quadro 1).

Quanto às ferramentas utilizadas nas intervenções, o sistema Nintendo Wii foi utilizado em onze estudos, dois estudos utilizaram o sistema Microsoft Kinect e nas ferramentas X-Box, 3D-ARS, DDR-Stepmania foram utilizados em apenas um estudo para cada ferramenta. Nas pesquisas selecionadas, pode-se observar que doze estudos realizaram dois tipos de protocolos. Um protocolo de intervenção foi realizado através de exercícios convencionais ou cinesioterapêuticos e compararam com um protocolo de intervenção associado ao uso de exergames.

Todos os artigos selecionados para a respectiva revisão sistemática caracterizam-se por serem jogos comerciais e não observou-se informações a respeito de jogos desenvolvidos (Quadro 1). 


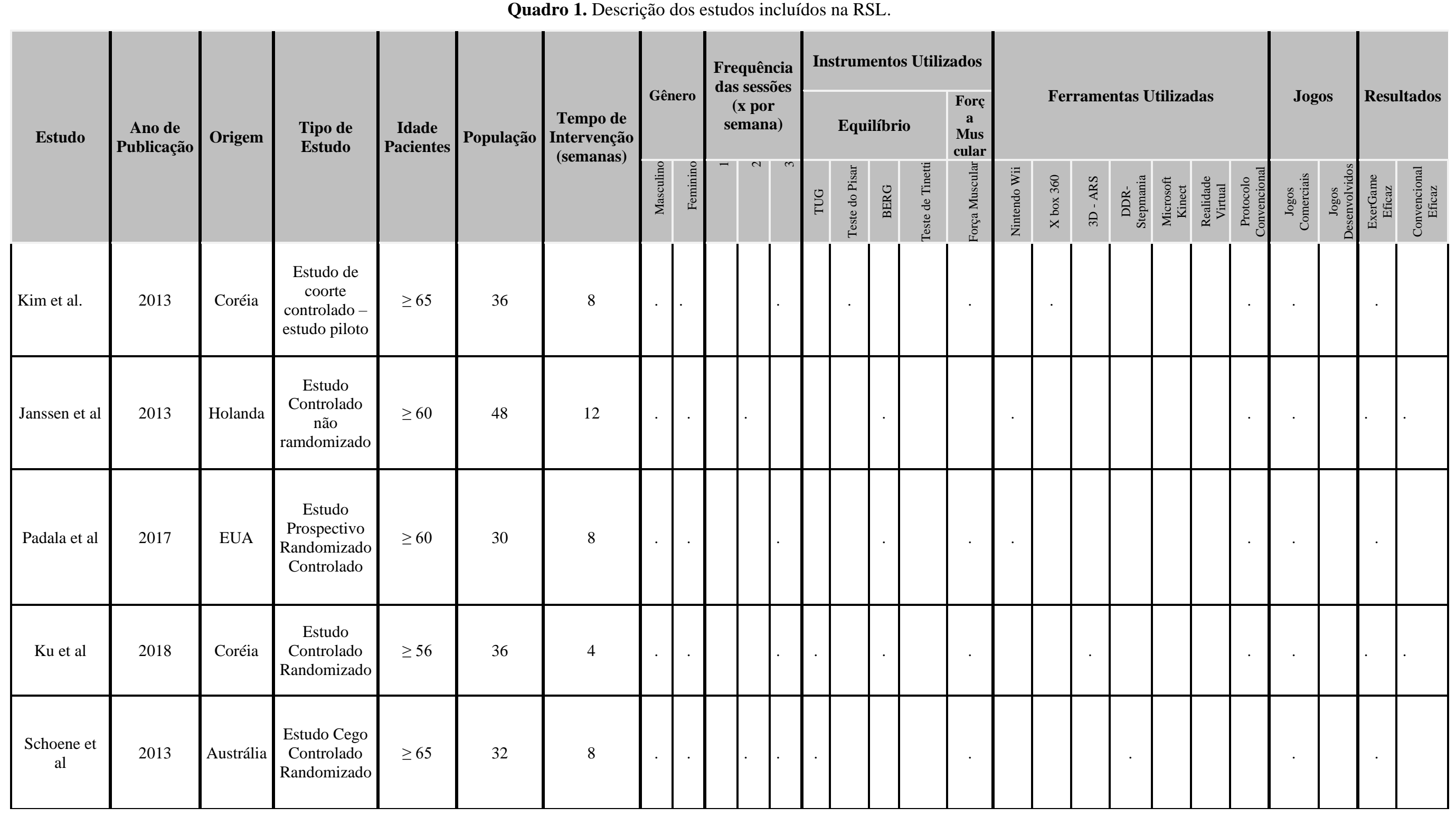


Research, Society and Development, v. 10, n. 13, e113101321063, 2021

(CC BY 4.0) | ISSN 2525-3409 | DOI: http://dx.doi.org/10.33448/rsd-v10i13.21063

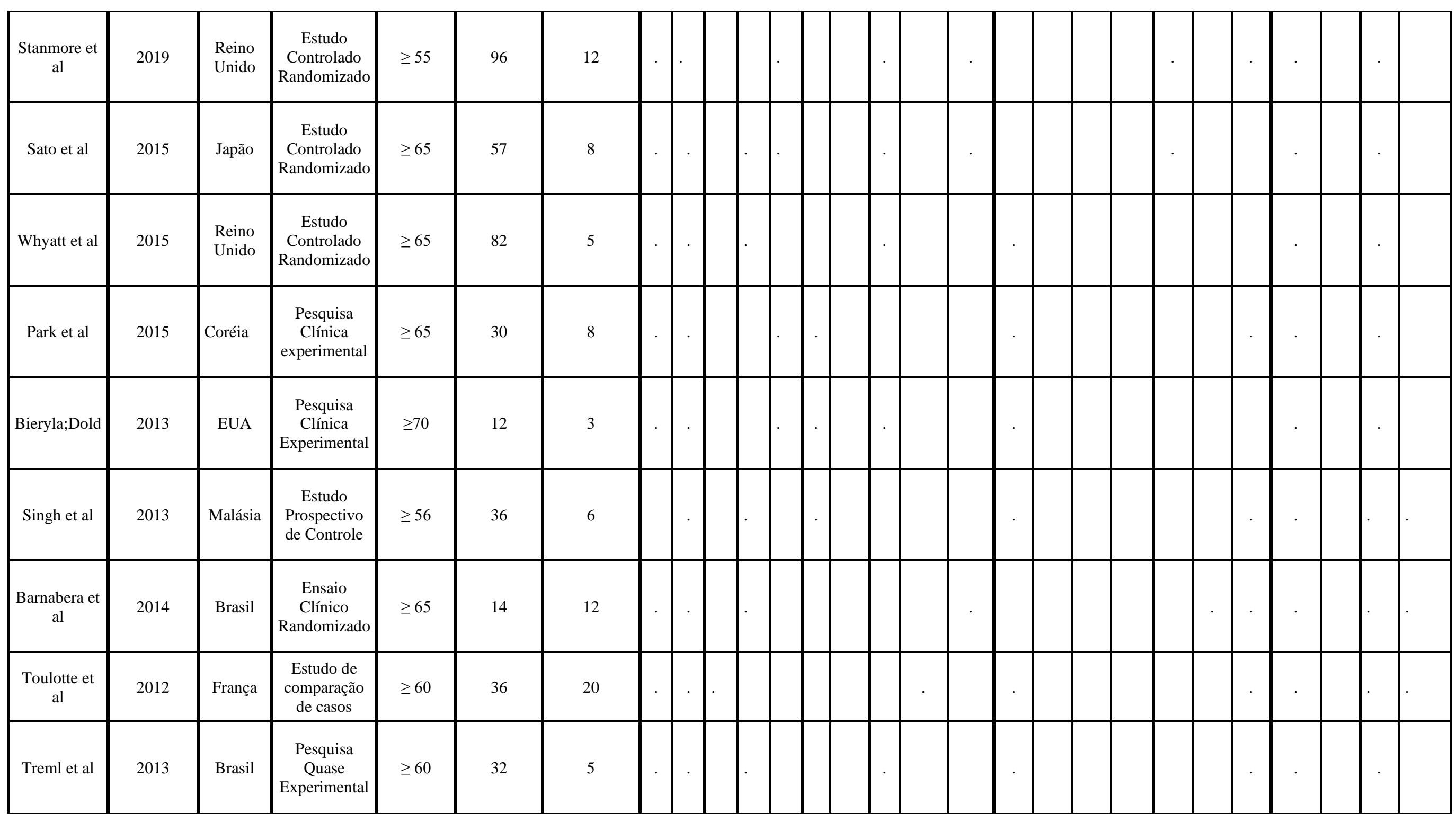


Research, Society and Development, v. 10, n. 13, e113101321063, 2021

(CC BY 4.0) | ISSN 2525-3409 | DOI: http://dx.doi.org/10.33448/rsd-v10i13.21063

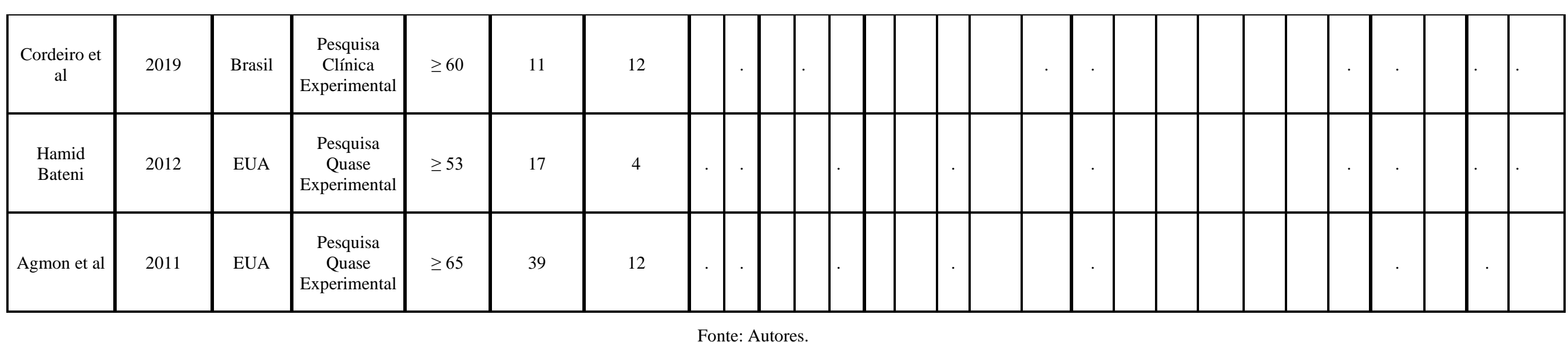


Em 10 estudos, os resultados foram positivos para os protocolos realizados com exergames (Tomicki et al., 2016; Agmon et al., 2011; Treml et al., 2013; Stanmore et al., 2019; Padala et al., 2017; Whyatt et al., 2015; Bieryla, 2013; Schoenen et al., 2012; Park et al., 2015; Jungjin et al., 2013) quando comparado com os protocolos convencionais. Nas pesquisas selecionadas (Barnabera et al., 2014; Ku et al., 2018; Cordeiro et al., 2019; Janssen et al., 2013; Bateni, 2012; Park et al., 2015; Singh et al., 2013) ambos os protocolos, intervenção associada ao exergame e intervenção através de um protocolo convencional, apresentaram resultados positivos.

Em relação a população, $82,35 \%$ das pesquisas selecionadas recrutaram tanto o gênero masculino quanto o feminino para a realização do estudo, totalizando quatorze estudos. A amostra do gênero feminino foi selecionada em apenas dois estudos, totalizando $11,77 \%$.

O número de frequência das sessões realizadas por semana também foi investigado. De acordo com os artigos selecionados, 8 deles $(47,05 \%)$ utilizaram uma frequência semanal de três vezes (Agmon et al., 2011; Ku et al., 2018; Stanmore et al., 2019; Padala et al., 2017; Bieryla, 2013; Bateni, 2012; Park et al., 2015; Jungjin et al., 2013). A frequência semanal de duas vezes foi observada em 6 estudos (35,29\%) (Barnabera et al., 2014; Treml et al., 2013; Cordeiro et al., 2019; Janssen et al., 2013; Whyatt et al., 2015; Singh et al., 2013). Dois estudos (Sato et al., 2015; Schoene et al., 2012) utilizaram uma frequência de duas a três vezes na semana, totalizando 11,76\%. Em apenas um estudo 27 os autores utilizaram uma frequência semanal de uma vez, totalizando $5,88 \%$, conforme a tabela 2.

Em relação ao número total de sessões, conforme a frequência semanal das pesquisas selecionadas, pode-se observar que dois estudos realizaram um protocolo com um número total de 36 sessões (Agmon et al., 2011; Stanmore et al., 2019). Os estudos que realizaram em seus protocolos um número total de 24 sessões totalizaram 35,2\% das pesquisas selecionadas, com 06 estudos (Barnabera et al., 2014; Padala et al., 2017; Cordeiro et al., 2019; Janssen et al., 2013; Park et al., 2015; Jungjin et al 2013). Os estudos de Sato et al. (2015) e Schoene et al. (2012) realizaram em suas pesquisas um número total de 16 a 24 sessões, conforme o número de sessões realizadas na semana. Um número de doze sessões no total do tempo de intervenção foi observado em três estudos (Ku et al., 2018; Bateni, 2012; Singh et al., 2013).

\section{Discussão}

Esta RSL incluiu 17 pesquisas com diferentes tipos de estudo, dentre os quais destacou-se o Estudo Controlado Randomizado. O objetivo desta revisão foi identificar e analisar estudos que abordaram os efeitos de um programa de intervenção cinesioterapêutica com exergame sobre a força muscular e equilíbrio em idosos saudáveis. Buscamos verificar também os métodos de avaliação do equilíbrio e força muscular utilizados e o tempo de intervenção realizado nas pesquisas selecionadas. A discussão foi baseada em quatro questões principais.

Pergunta-chave 1. Quais são os métodos de avaliação do equilíbrio e da força muscular usados?

Os testes de equilíbrio mais comumente utilizados foram a Escala de equilíbrio de Berg (EEB), com 10 estudos (Agmon et al., 2011; Treml et al., 2013; Ku et al., 2018; Stanmore et al., 2019; Padala et al., 2017; Janssen et al., 2013; Sato et al., 2015; Whyatt et al., 2015; Bieryla, 2013; Bateni, 2012) e o teste Time Up And Go (TUG), observado em 5 estudos (Ku et al., 2018; Bieryla, 2013; Schoene et al., 2012; Park et al., 2015; Singh et al., 2013; Albino et al., 2012). Esses resultados corroboram com os achados da revisão integrativa conduzida por Barbalho e Carvalho (2014), que identificaram as duas escalas com as mais descritas na literatura nacional e internacional para a avaliação segura do equilíbrio.

A Escala de Equilíbrio de Berg e o Teste Timed Up and Go foram validados, traduzidos, e adaptados para a língua portuguesa. São de fácil aplicação para a avaliação do equilíbrio. A Escala de equilíbrio de Berg (EEB) segundo Ku et al, (2018) é um instrumento validado e amplamente utilizado para testar habilidades de equilíbrio estático e dinâmico de um 
indivíduo. Compreende uma escala de 14 tarefas com cinco hipóteses de avaliação, chegando a um escore total de 0 a 56 pontos. É uma escala de fácil administração e é a mais aprimorada para detectar alterações no equilíbrio em idosos saudáveis (Cordeiro et al., 2019). Por se tratar de uma escala segura para a avaliação de idosos e por não necessitar de muitos materiais para a avaliação, justifica-se a opção dos autores na sua utilização. A escala Timed Up and Go avalia as alterações relacionadas a mobilidade funcional do equilíbrio dinâmico em idosos durante o desempenho de uma tarefa (Silva et al., 2008), e provavelmente essa questão tenha sido importante na escolha desta escala. Não foi encontrado nenhum estudo que relatasse que essas escalas devem ser usadas de maneira associada. Ambas as escalas podem ser utilizadas de forma individual na avaliação do equilíbrio de idosos (Ribeiro et al., 2005).

\section{Pergunta Chave 2. Qual o tempo total de intervenção utilizados nos estudos?}

O estudo de Toulotte et al (2012) foi o que apresentou o maior tempo de intervenção, com duração de 20 semanas. Nas pesquisas de (Barnabera et a., 2014; Agmon et al., 2011; Stanmore et al., 2019; Cordeiro et al., 2019 e Janssen et al., 2013) o tempo de intervenção foi de 12 semanas, totalizando $29,41 \%$ dos estudos incluídos. A mesma porcentagem foi observada em estudos que realizaram 08 semanas de intervenção (Padala et al., 2017; Sato et al., 2015; Schoene et al., 2012; Park et al., 2015; Jungjin et al., 2013). O tempo de intervenção de 06 semanas foi observado em apenas na pesquisa realizada por Singh et al (2013), totalizando 5,88\%. Em dois estudos selecionados, os autores Treml et al (2013) e Whyatt et al (2015) utilizaram 05 semanas de intervenção, totalizando 11,77\% dos estudos selecionados. Por sua vez, Bateni (2012) e Ku et al (2018) utilizaram um tempo de intervenção de 04 semanas (11,77\%). O menor tempo de intervenção foi de 3 semanas, proposto por Bieryla (2013). Independentemente da diferença entre o tempo de intervenção encontrado nos estudos incluídos, os autores concluíram que os protocolos realizados com o uso de exergame foram significativos na melhora do equilíbrio6, 7, 14, 15, 22, 23, 24, 25, $26,27,29,35$, bem como na melhora significativa da força muscular. 4, 13, 14, 15, 16, 22, 26, 29 Outra diferença em relação as pesquisas foi a frequência semanal das sessões. De todos os estudos selecionados, 94,12\% trabalharam com uma periodicidade mínima de frequência de duas a três vezes por semana, com exceção de Toulotte et al (2012) que optaram por trabalhar uma vez por semana, porém com tempo total de intervenção de 20 semanas. Na comparação entre as pesquisas, os resultados significativos apresentados pelos autores podem estar relacionados especificamente ao tempo de total de intervenção. Os autores utilizaram um protocolo de exercícios convencionais e a realização de exercícios com jogos comerciais na melhora do equilíbrio em idosos. Os resultados obtidos demonstraram ser estatisticamente significativos na melhora do equilíbrio em ambos os protocolos. Apesar de todos os estudos apresentarem tempos de intervenção e periodicidade semanal diferentes, todos demonstraram resultados significativos na melhora do equilíbrio, mesmo sendo um tempo de intervenção mínimo de apenas três semanas (Bieryla, 2012) e força muscular num período mínimo de quatro semanas em seus resultados. A partir do tempo mínimo de intervenção, pode-se perceber que os resultados já apresentaram-se positivos.

\section{Pergunta Chave 3. Quais os dispositivos utilizados?}

A utilização do Nintendo Wii® foi observada em 11 estudos, totalizando (64,70\%). Desses, Bateni 25, Park et al 27, Janssen et al 21, Singh et al 28 e Cordeiro et al 16 também usaram um protocolo convencional, sem o uso de qualquer tipo do jogo interativo. Bateni 25 e Cordeiro et al 16 avaliaram a força muscular de cada participante e os resultados não apresentaram diferença estatisticamente significativa entre os protocolos, sendo ambos efetivos na melhora da força muscular.

Andrade et al 31 com o uso do Nintendo Wiiß para o treinamento de equilíbrio de idosos institucionalizados, relataram que o tratamento inovou o mercado com um paradigma diferenciado, trazendo uma nova forma de jogar, com acessórios como o Balance Board e o jogo Wii Fit Plus. O objetivo foi de tornar a vida do jogador mais saudável, oferecendo 
jogos que estimulassem a realização de exercícios aeróbicos, equilíbrio e força muscular. Os autores ainda relataram que o Nintendo Wiiß pode gerar impacto positivo em idosos.

Janssen et al (2013), Singh et al (2013) e Park et al (2015) também utilizaram o Nintendo Wiiß através de um protocolo de exercícios e compararam com um protocolo de treinamento convencional. Os autores avaliaram o equilíbrio de idosos na pré e pós-intervenção. Os autores obtiveram resultados semelhantes entre os grupos que fizeram a intervenção com o Nintendo Wii ${ }^{\circledR}$ quando comparado com o grupo controle. Além do Nintendo Wii®, outros dispositivos foram usados. A realidade virtual é uma tecnologia que proporciona uma sensação de realidade através de feedback visual e auditivo (Jungjin et al., 2013). Os autores relataram que a realidade virtual através do X Box 360, pode tornar-se uma ferramenta acessível para idosos mais velhos, já que ela tem demonstrado eficácia na reabilitação, porém neste estudo não foi relatado qual tipo de dispositivo foi utilizado pelos autores. Jungjin et al (2013) e Stanmore et al (2019) avaliaram o equilíbrio e força muscular em idosos. Os dois estudos utilizaram ferramentas como jogos interativos e estes foram comparados com grupos que realizaram exercícios através de um protocolo convencional. Os autores concluíram que o grupo intervenção apresentou resultados melhores em relação ao grupo controle. Dessa forma, o exergame utilizado em ambos os estudos foi eficaz na melhora do equilíbrio e força muscular em idosos.

O Microsoft Kinect ${ }^{\circledR}$ foi utilizado nos estudos de Stanmore et al (2019) e Sato et al (2015) totalizando 11,76\% das pesquisas selecionadas. O console Xbox-360 foi observado em apenas um estudo de Jungjin et al (2013), assim como o 3DARS (Ku et al., 2018) e o DDR-Stepmania (Schoene et al., 2012). A reabilitação virtual foi citada apenas por um dos estudos selecionados (Barnabera et al., 2014). Os autores relataram o uso de jogos interativos através da reabilitação virtual com 3DARS. Os autores relataram que os exercícios da reabilitação virtual tornaram-se uma opção nos programas de reabilitação fisioterapêutica. Os resultados são positivos e incluem a melhora do equilíbrio, coordenação motora, resistência e força muscular, além do trabalho cognitivo. Nesta pesquisa os autores avaliaram e compararam os efeitos da reabilitação virtual e cinesioterapia, no torque dos músculos extensores do joelho de idosos saudáveis. Foram selecionados quatorze participantes e estes foram divididos em dois grupos aleatoriamente, o grupo reabilitação virtual e o grupo cinesioterapia. O protocolo de tratamento foi realizado no período de 12 semanas, com duas sessões semanais durante 50 minutos cada sessão. As atividades realizadas pelo grupo reabilitação virtual foram duas modalidades de jogos. $\mathrm{O}$ grupo cinesioterapia realizou os mesmos exercícios mas sem a utilização de qualquer jogo de realidade virtual. Os autores concluíram que após a intervenção ambos os grupos apresentaram melhora, mas sem diferença estatisticamente significativa entre os grupos e que a cinesioterapia assim como a reabilitação virtual são eficazes para o aumento do torque extensor de joelho em idosos saudáveis.

\section{Pergunta Chave 4. Quais estudos relacionaram as escalas de equilíbrio com protocolos com e sem o uso de exergames?}

Dos 10 estudos relacionados a avaliação através da EEB, Janssen et al (2013), Ku et al (2018) e Bateni (2012) compararam grupos que realizaram protocolos com exergames, e grupo que realizou atividades através de um protocolo de exercícios convencionais. Os resultados não demonstraram diferença significativa entre os grupos e ambos apresentaram melhora nos valores de equilíbrio na Escala de Berg. Em outras pesquisas, quando realizada a comparação entre grupos com o uso da Escala de Berg, Padala et al (2017), Stanmore et al (2019) e Treml et al (2013) concluíram que o grupo que realizou os exercícios com exergame apresentou melhora significativa em relação ao grupo do protocolo convencional.

Outras pesquisas realizadas por Agmon et al (2011), Sato et al (2015), Whyatt et al (2015), Bieryla (2013) que utilizaram a EEB, analisaram os efeitos de um protocolo de exercícios com exergames, antes e após o período de intervenção, sem grupo controle. Apesar do tempo de intervenção e da frequência semanal dos exercícios serem diferentes, os resultados obtidos foram que o treinamento com exergame foi eficaz na melhora do equilíbrio dos participantes. 
A avaliação com o teste Timed Get Up and Go (TUG) foi utilizada em cinco estudos (Ku et al., 2018; Bieryla, 2013; Schoene et al., 2012; Park et al., 2015; Singh et al., 2013) e teve como objetivo examinar a mobilidade funcional de idosos saudáveis. Esta avaliação é medida através do tempo em segundos, sendo necessário para executar as ações levantar-se de uma cadeira, caminhar três metros na velocidade de uma caminhada normal, virar-se, voltar e sentar novamente na cadeira. Dos cinco estudos selecionados que utilizaram a avaliação através do TUG, três deles compararam o uso de um protocolo com exercícios convencionais e um protocolo com uso de exergame. Ku et al (2018) e Singh et al (2013) relataram que ambos os protocolos foram significativos na melhora do teste TUG na avaliação de idosos. Park et al (2015) relataram que apenas o protocolo realizado através do exergame foi eficaz na melhora da avaliação através do TUG. Schoene et al (2012) e Bieryla (2013) utilizaram o protocolo de exercícios somente com a utilização de exergame e obtiveram resultados significativos na melhora do TUG no protocolo proposto.

Não foi verificado nenhum teste ou escala de equilíbrio Pisar (Junhgjin et al., 2013) nem o teste de Tinetti (Toulotte et al., 2012). Ambos os estudos objetivaram explorar a eficácia de um programa de exercício através de um exergame na melhora do equilíbrio em idosos. O teste de Tinetti mensura o equilíbrio estático e o equilíbrio dinâmico através de diferentes parâmetros da marcha, como a velocidade da caminhada, comprimento, distância, altura do degrau, bem como as mudanças de direção com os olhos fechados. Na pesquisa, os autores investigaram o uso de um exergame associado a um programa de atividades físicas em quatro diferentes grupos. Os resultados obtidos apontaram que os escores do teste de Tinetti diminuíram significativamente para os grupos que realizaram o programa de atividades físicas com e sem o uso de exergame. O teste de Pisar, relatado no estudo de Jungjin et al (2013), objetivou explorar a eficácia dos efeitos de um exergame no controle do equilíbrio em idosos. Todos esses estudos, ora mencionados, são importantes pois relatam sobre uma população que vem apresentando dentre algumas alterações, as músculo-esqueléticas. A diminuição do equilíbrio e da força muscular são em parte algumas das principais desordens encontradas no público idoso, principalmente naqueles que não tem o hábito de realizar exercícios físicos de forma regular. Por mais que as escalas de equilíbrio encontradas nas pesquisas selecionadas na RSL tenham sido diversas, todas elas objetivaram avaliar um programa de exercícios físicos na melhora do equilíbrio de idosos. O exercício físico é uma modalidade terapêutica que melhora o equilíbrio em idosos (Barbalho, 2014).

Limitações

Usamos termos de busca específicos, que podem não ter capturado todos os artigos sobre o assunto.

\section{Conclusão}

Esta revisão sistemática identificou 17 estudos na literatura em diferentes bases de dados. Os resultados mostraram uma variedade de instrumentos de avaliação, como a escala de equilíbrio de Berg e timed Up and Go e que o dispositivo mais utilizado nos estudos foi o Nintendo Wiiß, demonstrando que o protocolo de exercícios que o dispositivo proporciona é eficaz na melhora do equilíbrio e força muscular. O tempo de intervenção utilizado nas pesquisas foram díspares, variando de três a vinte semanas de tratamento. Com isso conclui-se que mesmo com diferenças entre o tempo de intervenção, através de um protocolo convencional quanto o protocolo através do exergame demonstraram resultados eficazes na melhora do equilíbrio e força em idosos saudáveis. Não foi observado nos estudos selecionados alguma pesquisa da qual os pesquisadores tivessem desenvolvido um game específico.

Espera-se que com a realização dessa Revisão Sistemática da Literatura, futuros estudos com o tema abordado possam ser realizados, principalmente no que diz respeito a avaliação da epigenética associada a realização de exercício físico associado a exergame. 


\section{Agradecimentos}

Os autores gostariam de agradecer à Fundação de Amparo à Pesquisa do Estado do Rio Grande do Sul (FAPERGS) e ao Conselho Nacional de Desenvolvimento Científico e Tecnológico (CNPq). Este estudo foi parcialmente financiado pela Coordenação de Aperfeiçoamento de Pessoal de Nível Superior - Brasil (CAPES) - Código Financeiro 001.

\section{Referências}

Davini, R., \& Nunes, C. V. (2003). Alterações no sistema muscular decorrentes do envelhecimento e o papel do exercício físico na manutenção da força muscular em idosos. Rev. Bras. Fisiot, 7(3), 201-207.

Tomicki, C., Zanini, S. C. C., Cecchin, L., Benedetti, T. R. B., Portella, M. R., Leguisamo, C. P. (2016). Efeito de um programa de exercícios físicos no equilíbrio e risco de quedas em idosos institucionalizados: ensaio clínico randomizado. Rev. Bras. Geriatr. Gerontol, 19(3), 473-482.

Manlapaz, D. G., Sole, G., Jayakaram, P. \& Chapple, C. M. (2017). A narrative synthesis of Nintendo wii fit gaming protocol in addressing balance among healthy older adults: what system works? Games For Health J, 6(2), 65-74.

Barnabera, M., Rodrigues, D. N., Cardoso, F. S., Marco, A. L., Francieli, P. M., Francica, J. V., Mazuchi, F. A. Z. \& Bigongiari, A. (2014). Estudo comparativo da reabilitação virtual e cinesioterapia em relação ao torque de joelho em idosos. Acta Fisiatr, 21(4), $171-176$.

Mendonça, C. S., Moura, S. K. M. S. F. \& Lopes, D.T. (2018). Benefícios do treinamento de força para idosos: revisão bibliográfica. Revista Campos do Saber, 4(1), 74-87.

Agmon, M., Perry, C. K., Phelan, E., Deminis, G. \& Nguyen, H. Q. (2011). A pilot study of wii fit exergames to improve balance in older adults. J Geriatr Phys Ther, 34(4), 161-167.

Treml, C. J., Filho, F. A. K., Cicarino, R. F. L., Wegner, R. S., Saita, C. Y. S. \& Corrêa, A.G. (2013). O uso da plataforma balance board como recurso fisioterápico em idosos. Rev. Bras. Geriatr. Gerontol, 16(4), 759-768.

Baracho, A. F. O., Gripp, F. J. \& Lima, M. R. (2012). Os exergames e a educação física escolar na cultura digital. Rev. Bras. Ciên. Esporte, $34(1)$, 111-126. Vilela, J. F., Soares, V. M. G. \& Maciel, A. M. S. B (2017). A importância prática da cinesioterapia em grupo na qualidade de vida de idosos. Acta Fisiatr, 24(3), 133-137.

Henrique, P. P. B., Colussi, E. L. \& De Marchi, A. C. (2019). Effects of Exergame on Patients' Balance and Upper Limb Motor Function after Stroke: A Randomized Controlled Trial. Journal of Stroke and Cerebrovascular Diseases, 28(8), 2351-2357.

Brandão, O. S., Neto, I.V. S., Rauber, S. B., Pimente, R. P. F. A., Zukowski, M. S., Fagundes, F. \& Campbell, C. S. G. (2017). Impacto de exergames na qualidade de diva em idosos. Fisioterapia Brasil, 18(3), 320-328.

Mussato, R., Brandalize, D. \& Brandalize, M. (2012). Nintendo Wii e seu efeito no equilíbrio e capacidade funcional de idosos saudáveis. R. Bras. Ci e Mov, $20(2), 68-75$.

Ku, J., Kim, Y. J., Cho, S., Lim, T., Lee, H. S. \& Kang, Y. J. (2018). Three-dimensional augmented reality system for balance and mobility rehabilitation in the elderly: a randomized controlled trial. Cyberpsychol Behav, 0(0), 1-10.

Stanmore, E. K., Mavroeidi, A., Jong, L. D., Skelton, D. A., Sutton, C. J., Benedetto, V., Munford, L. A., Meckes, W., Bell, V. \& Todd, C. (2019). The effectiveness and cost-effectiveness os strength and balance exergames to reduce falls risk people aged 55 years and older in UK assisted living facilities: a multicenter, cluster randomized controlled trial. BMC Medicine, 17(49), 1-14.

Padala, K. P., Padala, P. R., Lensing, S. Y., Dennis, R. A., Bopp, M. M., Parkes, C. M., Garrison, M. K., Dubbert, P. M., Roberson, P. K. \& Sullivan, D. H. (2017). Efficacy on wii-fit on static and dynamic balance in community dwelling older veterans: a randomized controlled pilot trial. Journal of aging Research, 2017, 1-9, doi: 10.1155/2017/465363.

Cordeiro, H. I. P., Rodrigues, A. C. M. A., Alves, M. R., Rojar, V. G., Maielot, P., Pimentel, D. M., Rezende, L. F., Rodrigues, V. D., Maia, M. F. M., Carneiro, L. S. F. \& Junior, R. S. M. (2019). Exercise with active video game or strength/balance training? Case reports comparing postural balance o folder women. Aging Clinical and Experimental Research, 10.1007/s40520-019-01219-1.

Wu, Z., Li, J. \& Theng, Y. L. (2015). Examining the Influencing Factors of Exercise Intention among Older Adults: A Controlled Study between Exergame and Traditional Exercise. Cyberpsychology, Behavior, and Social Networking, 18(9), 521-527.

Moher, D., Liberati, A., Tetzlaff, J. \& Altman, D. G. (2009). Preferred reporting items for systematic reviews and meta-analysis: the prisma statement. Plos Medicine, 6(7), DOI: 10.1371/Journal.pmed.1000097.g001.

NLM, Medical Subject Headings. DeCS - health sciences descriptors. Virtual health library. http://decs.bvs.br/.

Lo Russo, G., Spolveri, F., Ciancio, F. \& Mori, A. (2013). Mendeley: an easy way to manage, share, and synchronize papers and citations. Plast Reconstr Surg, 131(6), 946-947.

Janssen, S., Cange, H. \& Arends, R (2013). A preliminary study on the effectiveness of exergame Nintendo "wii fit plus" on the balance of nursing home residents. Games For Health J, 2(2), 89-95.

Sato, K., Kuroki, K., Saiki, S. \& Nagatomi, R. (2015). Improving walking, muscle strength and balance in the elderly with an exergame using Kinect: a randomized controlled trial. Games For Health J, 4(3), 161-167. 
Whyatt, C., Merriman, N. A., Young, W. R., Newell, F. N. \& Craig, C. (2015). A wiibit of fun: a novel plataform to deliver effective balance training to older adults. Games For Health J, 4(6), 423-433.

Bieryla, K. A. \& Dold, N. M. (2013). Feasibility of wii fit training to improve clinical measures of balance in older adults. Clinical Interventions in Aging, 8 , 775-781.

Bateni, H. (2012). Changes in balance in older adults based on use of physical therapy vs the fit gaming system: a preliminary study. Physiotherapy, 98(3), 211-216.

Schoene, D., Lord, S, R., Severino, C., Davies, T. A. \& Smith, S. T. (2013). A randomized controlled pilot study of home-based step training in older people using videogame technology. Plos One, 8(3), 1-8.

Park, E. C., Kim, S. G. \& Lee, C. W. (2015). The effects of virtual reality game exercise on balance and gait of the elderly. J Phys. Ther. Sci, 27(4), 11571159 .

Singh, D. K. A., Rajaratnam, B. S., Palaniswaniy, V., Raman, V. P., Bong, P. S. \& Pearson, H. (2013). Effects of balance-focused interactive games compared to therapeutic balance classes for older adults. Climateric, 16(1), 141-146.

Jungjin, K., Son, J., Ko, N. \& Yoon, B. (2013). Unsupervised virtual reality-based exercise program improves hip muscle strength and balance control in older adults: a pilot study. Archives oh Physical Medicine and Rehabilitation, 94(5), 937-943.

Albino, I. L. R., Freitas, C. I. R., Teixeira, A. R., Gonçalves, A. K., Santos, A. M. P. V. \& Bós, A. J. G. (2012). Influência do treinamento de força muscular e de flexibilidade articular sobre o equilíbrio corporal em idosas. Rev. Bras. Geriatr. Gerontol, 15(1), 17-25.

Andrade, E. C. S., Melo, W., Dini, P. D. D. \& Pinheiro, H. Á. (2013). A utilização do Nintendo wii no treinamento de equilíbrio de idosos: estudo piloto. Fisioterapia Brasil, 14(4), 264-267.

Barbalho, E. V. \& Carvalho, A. D. F. (2014). Métodos e protocolos de avaliação do equilíbrio: uma revisão. Sobral, 1(4), 01-16.

Silva, A., Almeida, G. J. M., Cassilhas, R. C., Cohen, M., Peccin, M. S., Tufik, S. \& Mello, M. T. (2008). Equilíbrio, coordenação e agilidade de idosos submetidos à prática de exercícios físicos resistidos. Rev Bras Med Esporte, 14(2), 88-93.

Ribeiro, A. S. B. \& Pereira, J. S. (2005). Melhora do equilíbrio e redução da possibilidade de quedas em idosas após exercícios de cawthorne e cooksey. Rev Bras Otorrinolaringol, 71(1), 38-46.

Toulotte, C., Toursel, C. \& Olivier, N. (2012). Wii Fit training vs. Adapted physical activities: wich one is the most appropriate to improve the balance of independent senior subjects? A randomized controlled study. Clinical Rehabilitation, 26(9), 827-835. 